\title{
Large Scale Structure in Bekenstein's Theory of Relativistic Modified Newtonian Dynamics
}

\author{
C. Skordis, ${ }^{1}$ D. F. Mota, ${ }^{2}$ P. G. Ferreira, ${ }^{1,3}$ and C. Bœhm ${ }^{4,5}$ \\ ${ }^{1}$ Astrophysics, University of Oxford, DWB, Keble Road, Oxford OX1 3RH, United Kingdom \\ ${ }^{2}$ Institute for Theoretical Astrophysics, University of Oslo, N-0315 Oslo, Norway \\ ${ }^{3}$ African Institute for Mathematical Sciences (AIMS), 6-8 Melrose Road, Muizenberg 7945, South Africa \\ ${ }^{4}$ TH Division, PH Department, CERN 1211, Geneve 23, Switzerland \\ ${ }^{5}$ LAPTH, UMR 5108, 9 chemin de Bellevue -BP 110, 74941 Annecy-Le-Vieux, France
}

(Received 25 May 2005; published 3 January 2006)

\begin{abstract}
A relativistic theory of modified gravity has been recently proposed by Bekenstein. The tensor field in Einstein's theory of gravity is replaced by a scalar, a vector, and a tensor field which interact in such a way to give modified Newtonian dynamics (MOND) in the weak-field nonrelativistic limit. We study the evolution of the Universe in such a theory, identifying its key properties and comparing it with the standard cosmology obtained in Einstein gravity. The evolution of the scalar field is akin to that of tracker quintessence fields. We expand the theory to linear order to find the evolution of perturbations on large scales. The impact on galaxy distributions and the cosmic microwave background is calculated in detail. We show that it may be possible to reproduce observations of the cosmic microwave background and galaxy distributions with Bekenstein's theory of MOND.
\end{abstract}

DOI: 10.1103/PhysRevLett.96.011301

PACS numbers: 98.80.Cq, 98.65.Dx, 98.70.Vc

The current model of the Universe is based on a few simple assumptions and can explain a multitude of observation. Yet, to be able to explain the structure of galaxies and clusters of galaxies, it is essential to postulate the existence of some invisible substance, called dark matter. Although there are reports of tentative discoveries of dark matter [1], there is no proven theory or direct observation of a dark matter particle as yet. A less explored route is that our current theory of gravity might be incomplete. Given that the existence of dark matter is inferred from its gravitational effects on the dynamics of astrophysical bodies, it may be that the theory used to link the dynamics to the mass is incorrect. Milgrom has proposed a modification of Newtonian dynamics, known as MOND [2]. In MOND, Newton's second law in a gravitational field is modified to $\mu\left(|\vec{a}| / a_{0}\right) \vec{a}=-\nabla \Phi$ where $\vec{a}$ is the acceleration, $\Phi$ is the Newtonian potential, and $\mu(x)$ is a function with a scale $x \simeq 1$. For $|\vec{a}|>a_{0}$ we have that $\mu(x)=1$. With $|\vec{a}|<a_{0}$ we have that $\mu(x) \simeq x$. Clearly for small accelerations, Newtonian theory is no longer valid. Milgrom's theory has been extremely successful in explaining a number of observational properties of galaxies [3]. It has suffered from a fatal flaw in that it is not generally covariant and hence cannot be studied in a general setting.

Bekenstein has recently solved this problem [4]. Building on a series of developments [5], he has proposed a generally covariant theory which has in the nonrelativistic, weak-field limit, Milgrom's modified theory. Bekenstein's theory has two metrics. One of the metrics, $\tilde{g}_{\mu \nu}$ has its dynamics governed by the Einstein-Hilbert action,

$$
S_{g}=\frac{1}{16 \pi G} \int d^{4} x \sqrt{-\tilde{g}} \tilde{R},
$$

where $G$ is Newton's constant and $\tilde{R}$ is the scalar curvature of $\tilde{g}_{\mu \nu}$. We shall call the frame of this metric the "Einstein frame" (EF). The second metric, $g_{\mu \nu}$ is minimally coupled to all the matter fields in the Universe. We shall call the frame of this metric the "matter frame" (MF). All geodesics are calculated in terms of this second metric. The two metrics are related through $g_{\mu \nu}=e^{-2 \phi}\left(\tilde{g}_{\mu \nu}+\right.$ $\left.A_{\mu} A_{\nu}\right)-e^{2 \phi} A_{\mu} A_{\nu}$. Two fields are required to connect the two metrics. The scalar field, $\phi$ has dynamics given by the action

$S_{s}=-\frac{1}{16 \pi G} \int d^{4} x \sqrt{-\tilde{g}}\left[\mu\left(\tilde{g}^{\mu \nu}-A^{\mu} A^{\nu}\right) \phi_{, \mu} \phi_{, \nu}+V(\mu)\right]$,

where $\mu$ is a nondynamical field and $V$ is a free function which can be chosen to give the correct nonrelativistic MOND limit and depends on two free parameters, $l_{B}$ and $\mu_{0}$ (related to $\kappa$ in [4] as $\mu_{0}=8 \pi / \kappa$ ). The unit timelike vector field, $A_{\mu}$ has dynamics given by the action

$$
S_{v}=-\frac{1}{32 \pi G} \int d^{4} x \sqrt{-\tilde{g}}\left[K F^{\alpha \beta} F_{\alpha \beta}-2 \lambda\left(A^{\mu} A_{\mu}+1\right)\right],
$$

where $F_{\mu \nu}=A_{\mu, \nu}-A_{\nu, \mu}$, indices are raised with $\tilde{g}$ and where $K$ is the third parameter in this theory. The Lagrange multiplier $\lambda$ is completely fixed by variation of the action.

We wish to study the evolution of a homogeneous and isotropic universe in such a theory. Observers are defined in the MF where the line element is $d s^{2}=a^{2}\left(-d \eta^{2}+d r^{2}\right)$. The scale factor is related to the metric in the EF, $d \tilde{s}^{2}=$ $b^{2}\left(-e^{-4 \phi} d \eta^{2}+d r^{2}\right)$ through $a=b e^{-\phi}$. The modified Friedmann equation becomes

$$
3 \frac{\dot{b}^{2}}{b^{2}}=a^{2}\left[\frac{1}{2} e^{-2 \phi}\left(\mu V^{\prime}+V\right)+8 \pi G e^{-4 \phi} \rho\right],
$$


where $\mu$ can be found by inverting $\dot{\phi}^{2}=\frac{1}{2} a^{2} e^{-2 \phi} \frac{d V}{d \mu}$ and the energy density $\rho$ does not include $\phi$. Homogeneity and isotropy and the constraint in the action imply that $A_{\mu}$ is fixed as $A_{\mu}=a e^{-\phi}(1,0,0,0)$. The background dynamics is complete with an equation for $\phi$ :

$$
\begin{aligned}
\ddot{\phi}= & -a^{2} e^{-2 \phi} V^{\prime}-\frac{1}{U}\left[2\left(\mu-\frac{V^{\prime}}{V^{\prime \prime}}\right) \frac{\dot{b}}{b} \dot{\phi}\right. \\
& \left.+4 \pi G a^{2} e^{-4 \phi}(\rho+3 P)\right],
\end{aligned}
$$

where $U=\mu+2 V^{\prime} / V^{\prime \prime}$ and, once again, $P$ does not include the pressure from the scalar field $\phi$.

A choice of $V$ will pick out a given theory. As a first guess, Bekenstein has proposed

$$
V=\frac{3 \mu_{0}^{2}}{128 \pi l_{B}^{2}}\left[\hat{\mu}\left(4+2 \hat{\mu}-4 \hat{\mu}^{2}+\hat{\mu}^{3}\right)+2 \ln (\hat{\mu}-1)^{2}\right],
$$

where $\hat{\mu}=\mu / \mu_{0}$. This potential will lead to the prescription proposed by Milgrom in the nonrelativistic region. The evolution of this coupled set of equations is essentially insensitive to $l_{B}$ and independent of $K$ but is well determined in terms of $\mu_{0}$. If we define the physical Hubble parameter, $H$ we can rewrite the modified Friedmann equations in the form $3 H^{2}=8 \pi G_{\text {eff }}\left(\rho+\rho_{\phi}\right)$ where the effective Newton's constant has the form $G_{\text {eff }}=G e^{-4 \phi} /$ $\left(1+\frac{d \phi}{d \ln a}\right)^{2}$ and the energy density in $\phi$ is

$$
\rho_{\phi}=\frac{e^{2 \phi}}{16 \pi G}\left(\mu V^{\prime}+V\right)
$$

This system exhibits the tracking behavior witnessed in some scalar field theories of quintessence [6]. In particular, one finds that for a wide range of initial conditions, $\phi$ evolves to a slowly varying function of time and the relative energy density in $\phi$ reaches an attractor solution of the form $\Omega_{\phi}=3 /\left(2 \mu_{0}\right)$ in the radiation era and $\Omega_{\phi}=$ $1 /\left(6 \mu_{0}\right)$ in the matter and $\Lambda$ eras.

In Fig. 1 we solve the equations numerically for a low value of $\mu_{0}$ to illustrate the tracking behavior of $\phi$. As with

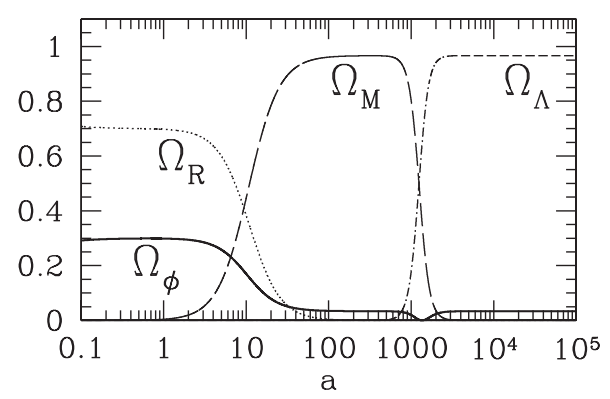

FIG. 1. The relative energy densities in $\phi$ (thick solid line), radiation (dotted line), matter (dashed line), and $\Lambda$ (dot-dashed line) for $\mu_{0}=5$ as a function of the scale factor ( $a$ is in arbitrary units). Note that the energy density in the scalar field tracks the dominant form of energy at each instance in time. other tracking systems we can constrain the energy density in $\phi$ at nucleosynthesis [7]. The abundance of light elements is extremely sensitive to the expansion rate at $1 \mathrm{MeV}$ and can, for example, be used to constrain the number of relativistic degrees of freedom at that time. Recent measurements of the ${ }^{4} \mathrm{He}$ mass fraction and the deuterium abundance leads to a bound at energies of $1 \mathrm{MeV}$ of $\Omega_{\phi}<0.045$ at the $95 \%$ confidence level. This leads to $\mu_{0}>33$ and hence the scalar field will make up less than $0.5 \%$ of the total energy density during the matter and $\Lambda$ dominated eras.

We now turn to evolution of linear perturbations on this background. This will allow us to link this theory with observations of galaxy clustering on large scales as well as with the anisotropies in the cosmic microwave background (CMB). The main problem that a MOND theory containing only baryons has to confront is the damping of perturbations during the recombination era. Indeed in a pure baryonic universe evolving under Einstein gravity, the weak coupling of baryons and photons during the recombination era will lead to Silk damping, the collisional propagation of radiation from overdense to underdense regions [8]. In the standard adiabatic model with just baryons, the matter power spectrum is severely supressed on galactic scales. If MOND is to succeed, it must overcome the Silk damping on these scales.

There are three fields to perturb around the background (we identify the homogeneous part of a quantity $X$ by $\bar{X}$ ). The MF metric perturbations are given in the conformal Newtonian gauge by $g_{00}=-a^{2}(1+2 \Psi)$ and $g_{i j}=$ $a^{2}(1-2 \Phi) \delta_{i j}$. The scalar field perturbation is given by $\phi=\bar{\phi}+\varphi$. The vector field perturbation is defined by $A_{\mu}=a e^{-\bar{\phi}}\left(\bar{A}_{\mu}+\alpha_{\mu}\right)$ where the scalar components, $\alpha$ and $E$, of $\alpha_{\mu}=(\Psi-\varphi, \vec{\alpha})$ are given by $\nabla^{2} \alpha \equiv \nabla \cdot \vec{\alpha}$ and $\nabla^{2} E \equiv \nabla \cdot \vec{E}$ where we use the field strength tensor of $A_{\mu}, F_{\mu \nu}$ to define the "electric field" through $E_{i}=$ $\bar{A}^{\mu} F_{i \mu}$. Similarly, in EF, we have $\tilde{g}_{00}=-b^{2} e^{-4 \bar{\phi}}(1+$ $2 \tilde{\Psi}), \tilde{g}_{0 i}=-b^{2} \tilde{\zeta}_{, i}$, and $\tilde{g}_{i j}=b^{2}(1-2 \tilde{\Phi}) \delta_{i j}$ which give $\tilde{\Psi}=\Psi-\varphi, \tilde{\Phi}=\Phi-\varphi$, and $\tilde{\zeta}=-\left(1-e^{-4 \bar{\phi}}\right) \alpha$.

The evolution equations for the matter fluid remain unaltered if expressed in terms of the MF variables. That is if we expand densities as $\rho=\bar{\rho}(1+\delta)$ and use the standard definition for momentum of the fluid, $\nabla^{2} \theta=\nabla$. $\vec{v}$, the evolution equations remain the same as in Einstein gravity. Two new sets of evolution equations must be introduced. For the scalar field perturbations, we have

$$
\begin{aligned}
\dot{\varphi}= & -\frac{a e^{-\bar{\phi}}}{2 U} \gamma+\dot{\bar{\phi}} \tilde{\Psi} \\
\dot{\gamma}= & -3 \frac{\dot{b}}{b} \gamma+\frac{\bar{\mu}}{a} e^{-3 \bar{\phi}} k^{2}(\varphi+\dot{\bar{\phi}} \alpha)-2 \frac{e^{\bar{\phi}}}{a}\left(3 \dot{\tilde{\Phi}}+k^{2} \tilde{\zeta}\right) \\
& +8 \pi G a e^{-3 \bar{\phi}} \bar{\rho}\left[\left(1+3 c_{s}^{2}\right) \delta+(1+3 w)(\tilde{\Psi}-2 \varphi)\right],
\end{aligned}
$$

and for the vector field we have 


$$
\begin{aligned}
\dot{\alpha}= & \frac{e^{\bar{\phi}}}{a} E+\tilde{\Psi}+\left(\dot{\bar{\phi}}-\frac{\dot{a}}{a}\right) \alpha, \\
K \frac{e^{2 \bar{\phi}}}{a^{2}}(\dot{E}+2 \dot{\bar{\phi}} E)= & -\mu \frac{e^{\bar{\phi}}}{a} \dot{\bar{\phi}}(\varphi-\dot{\bar{\phi}} \alpha)+16 \pi G a e^{-\bar{\phi}} \\
& \times \sinh (2 \bar{\phi})(1+w) \bar{\rho}(\theta-\alpha),
\end{aligned}
$$

where $w=\bar{P} / \bar{\rho}$ and $c_{s}^{2}=\delta P / \delta \rho$. The perturbed Einstein equations allow us to identify the gravitational potentials through

$$
\begin{aligned}
2 k^{2} \tilde{\Phi}= & -2 e^{4 \bar{\phi}} \frac{\dot{b}}{b} k^{2} \tilde{\zeta}-e^{4 \bar{\phi}} \dot{\bar{\phi}}\left\{-a e^{-\bar{\phi}} \gamma+6 \bar{\mu} \frac{\dot{b}}{b} \varphi\right\} \\
& -8 \pi G a^{2} \bar{\rho}\left\{\delta+3(1+w) \frac{\dot{b}}{b} \theta-2 \varphi\right\}-K k^{2} \frac{e^{\bar{\phi}}}{a} E, \\
\dot{\tilde{\Phi}}= & 4 \pi G a^{2} e^{-4 \bar{\phi}}(1+w) \bar{\rho} \theta+\bar{\mu} \dot{\bar{\phi}} \varphi-\frac{\dot{b}}{b} \tilde{\Psi}, \\
\tilde{\Psi}= & \tilde{\Phi}+e^{4 \bar{\phi}}\left[\dot{\tilde{\zeta}}+2\left(\frac{\dot{b}}{b}+\dot{\bar{\phi}}\right) \tilde{\zeta}\right]-\frac{12 \pi G}{k^{2}} a^{2}(1+w) \rho \sigma,
\end{aligned}
$$

where $\sigma$ is the total shear from matter fluids.

We have modified CMBEASY, a publicly available numerical Einstein-Boltzmann solver to incorporate the modified background and perturbation equations [9]. The evolution equations have been implemented in both the conformal Newtonian and synchronous gauge to check for consistency [10]. We have restricted ourselves to a flat universe with a cosmological constant but considered the possibility of massive neutrinos.

The parameters $\mu_{0}, K$, and $l_{B}$ may introduce major modifications in the morphology of perturbations. A low $\mu_{0}$, low $l_{B}$, and low $K$ will lead to a change in the growth rate. As we can see in Fig. 2, the effect is to introduce an integrated Sachs-Wolfe term which can be quite significant. For example, for sufficiently small $l_{B}$, the structure of the angular power spectrum of the CMB can be completely modified with an excess of large scale power overwhelming structure on the smallest scales. We can see the effect of modifying $l_{B}$ in the middle panel of Fig. 2. Clearly, the $\mathrm{CMB}$ can place quite stringent constraints on the values of these parameters. A further possible effect, that we have not included here, is the effect of secondary anisotropies such as the Sunyaev-Zel'dovich or Ostriker-Vishniac effects, which may leave a difference signature than the standard cold dark matter $\Lambda$ CDM model.

Since the baryon content is set by the abundance of light elements, we must compensate with a high value of the cosmological constant, i.e., with $\Omega_{\Lambda} \simeq 0.95$. An obvious consequence of this is that the angular-distance relation will be modified as compared to the standard adiabatic $\Lambda \mathrm{CDM}$ universe [11]. Indeed the position of the peaks in the angular power spectrum of the CMB will be shifted to higher $l \mathrm{~s}$ which would lead to a severe mismatch with the current available data from the Wilkinson Microwave Anisotropy Probe and other experiments. A natural solution to this is to include a small component of massive

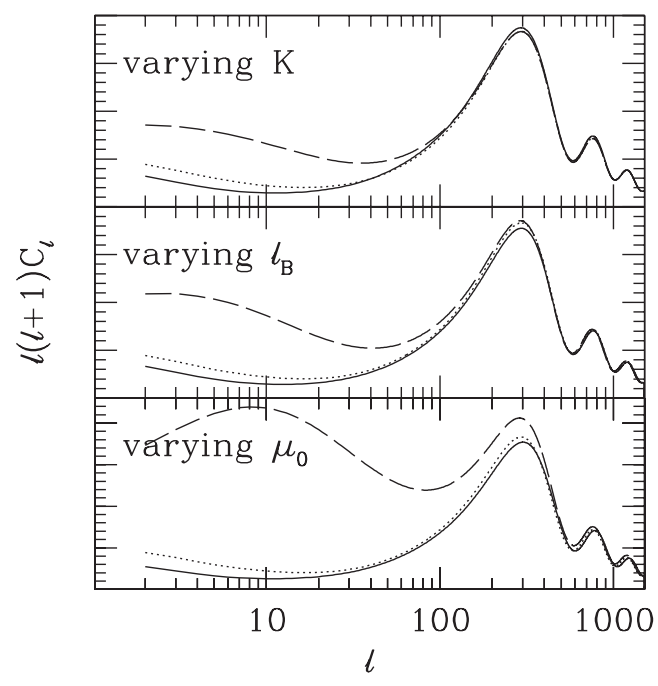

FIG. 2. The effect of the MOND parameters on the power of spectrum of the CMB. Top panel: $\mu_{0}=200, l_{B}=100 \mathrm{Mpc}$, and $K=1$ (solid), 0.1 (dotted), and 0.08 (dashed); Middle panel: $\mu_{0}=200, K=0.1$, and $l_{B}=1000 \mathrm{Mpc}$ (solid), $100 \mathrm{Mpc}$ (dotted), and $10 \mathrm{Mpc}$ (dashed); Bottom panel: $K=0.1, l_{B}=$ $100 \mathrm{Mpc}$, and $\mu_{0}=1000$ (solid), 200 (dotted), and 150 (dashed).

neutrinos, $\Omega_{\nu} \simeq 0.15$. As we can see in the top panel of Fig. 4, with this modification we can reproduce the temperature anisotropy data.

The main question we have raised is whether MOND dynamics can inhibit the damping of small scale perturbations in the coupled baryon-photon fluid during recombination. Recall that in the adiabatic CDM model, perturbations in the dark matter, $\delta_{C}$, are undamped during recombination. The Newtonian potential, which is roughly given by $k^{2} \Phi \simeq 4 \pi G\left(\rho_{B} \delta_{B}+\rho_{C} \delta_{C}\right)$ will not be erased if $\rho_{C}$ is sufficiently large, even though $\delta_{B} \rightarrow 0$ through recombination [12]. In the MOND universe we find an analogous effect; we now have $k^{2} \Phi \simeq 4 \pi G \rho_{B}\left(\delta_{B}-2 \varphi\right)$. The perturbation in the scalar field will support the perturbations through recombination yet still allow the damping of anisotropies in the photon fluid. Unlike the case of dark matter however, the coupling between the scalar field and the metric is such that $\rho_{\phi}$ does not play a role in the magnitude of the effect. Even for minute values of $\Omega_{\phi}$ we can still have a non-negligible effect. As we can see in Fig. 3, the net result is that decreasing $\mu_{0}, l_{B}$, or $K$ will boost small scale power in such a way as to overcome the damping of perturbations. This is an intriguing effect that goes in tandem with what we saw in the CMB. While decreasing $l_{B}$ (and a sufficiently small $K$ and $\mu_{0}$ ) will contaminate the large scale power in the angular power spectrum of the CMB, it can also play a role in counteracting Silk damping of density perturbations.

Given these two effects on the dynamics of large scale structure, is it possible to construct a MOND universe which can reproduce current observations of the CMB and galaxy surveys? There is clearly a competition be- 


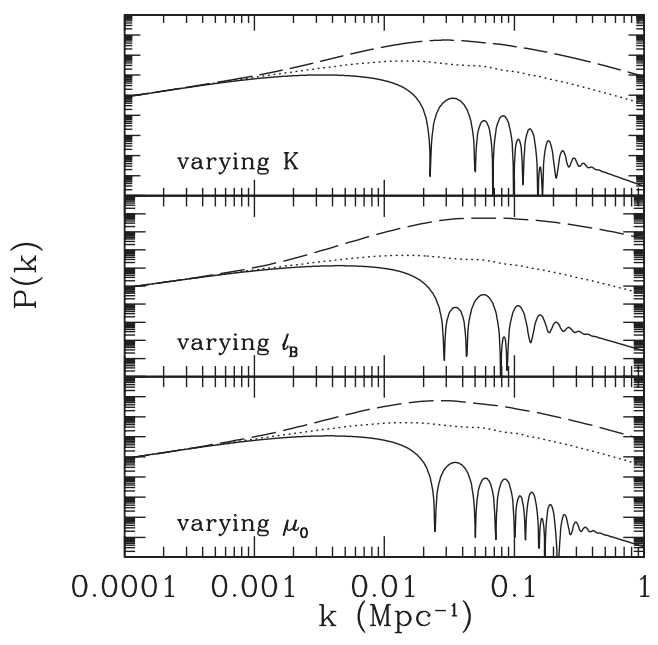

FIG. 3. The effect of the MOND parameters on the power of spectrum of the baryonic density fluctuations. Top panel: $\mu_{0}=$ 200, $l_{B}=100 \mathrm{Mpc}$, and $K=1$ (solid), 0.1 (dotted), and 0.08 (dashed); Middle panel: $\mu_{0}=200, \quad K=0.1$, and $l_{B}=$ $1000 \mathrm{Mpc}$ (solid), $100 \mathrm{Mpc}$ (dotted), and $10 \mathrm{Mpc}$ (dashed); Bottom panel: $K=0.1, l_{B}=100 \mathrm{Mpc}$, and $\mu_{0}=1000$ (solid), 200 (dotted), and 150 (dashed).

tween overproducing large scale power in the CMB but also overcoming damping on small scale. In Fig. 4 we present two MOND universes compared to data [13,14]. As mentioned above, a universe with a very large contribution of $\Lambda$ will not fit the current CMB data. By having the three neutrinos with a mass of $m_{\nu} \simeq 2 \mathrm{eV}$ each we are able to resolve this mismatch. With an appropriate choice of $K, \mu_{0}$, and $l_{B}$ it is possible to reproduce the power spectrum of galaxies as inferred from the Sloan digital
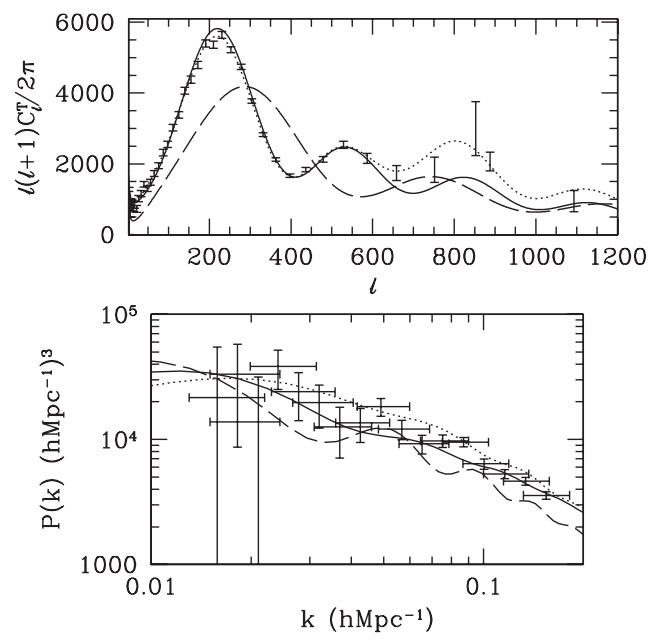

FIG. 4. The angular power spectrum of the CMB (top panel) and the power spectrum of the baryon density (bottom panel) for a MOND universe (with $a_{0} \simeq 4.2 \times 10^{-8} \mathrm{~cm} / \mathrm{s}^{2}$ ) with $\Omega_{\Lambda}=$ 0.78 and $\Omega_{\nu}=0.17$ and $\Omega_{B}=0.05$ (solid line), for a MOND universe $\Omega_{\Lambda}=0.95$ and $\Omega_{B}=0.05$ (dashed line) and for the $\Lambda C D M$ model (dotted line). A collection of data points from CMB experiments and Sloan are overplotted. sky survey [14]. The possibility of using massive neutrinos to resolve some of the problems with clusters in a MOND universe has been mooted in [15].

We have focused on one very specific model proposed by Bekenstein with a somewhat artificial potential for the new degrees of freedom. This phenomenological approach needs a firmer theoretical underpinning which might come from the various approaches which are being taken in the context of brane worlds, $M$ theory, and a rich array of theories of modified gravity. However, Bekenstein's theory can play an important role in opening up an altogether different approach to the dark matter problem. It serves as a proof of concept which will clearly lead to a new, very different view of the role played by the gravitational field in cosmology.

We thank J. Bekenstein, J. Binney, M. Doran, J. Dunkley, O. Elgaroy, J.-M. Frere, D. Hooper, S. Pascoli, and O. Vives for discussions. C. S. is supported by PPARC Grant No. PPA/G/O/2001/00016. D. F. M. is supported by the Research Council of Norway through Project No. $159637 /$ V30.

[1] G. Bertone, D. Hooper, and J. Silk, Phys. Rep. 405, 279 (2004).

[2] M. Milgrom, Astrophys. J. 270, 365 (1983); 270, 371 (1983); 270, 384 (1983).

[3] R. Sanders and S. McGaugh, Annu. Rev. Astron. Astrophys. 40263 (2002); S. McGaugh and E. de Blok, Astrophys. J. 499, 66 (1998).

[4] J. D. Bekenstein, Phys. Rev. D 70, 083509 (2004);

[5] J.D. Bekenstein and Milgrom, Astrophys. J. 286, 7 (1984); J. D. Bekenstein, Phys. Lett. B 202, 497 (1988); R. H. Sanders, Astrophys. J. 480, 492 (1997).

[6] B. Ratra and P. J. Peebles, Phys. Rev. D 37, 3406 (1988); C. Wetterich, Nucl. Phys. B252, 302 (1988); E. Copeland et al., Ann. N.Y. Acad. Sci. 688, 647 (1993); P. G. Ferreira and M. Joyce, Phys. Rev. Lett. 79, 4740 (1997); C. Skordis and A. Albrecht, Phys. Rev. D 66, 043523 (2002).

[7] R. Bean, S. Hansen, and A. Melchiorri, Phys. Rev. D 64, 103508 (2001).

[8] P. J. Peebles and J. T. Yu, Astrophys. J. 162, 815 (1970); M. L. Wilson and J. Silk, Astrophys. J. 243, 14 (1981); L. Griffiths, A. Melchiorri, and J. Silk, Astrophys. J. 553, L5 (2001).

[9] M. Doran, http://www.arxiv.org/ astro-ph/0302138.

[10] C. Skordis, astro-ph/0511591.

[11] G. Efstathiou and D. Bond, Mon. Not. R. Astron. Soc. 304, 75 (1999).

[12] P. J. Peebles, Astrophys. J. 248, 885 (1981).

[13] C. Bennett et al. (WMAP collaboration), Astrophys. J. Supp. 148, 1 (2003); X. Wang et al., Phys. Rev. D 68, 123001 (2003); C.L. Kuo et al., Astrophys. J. 600, 32 (2004); J. Ruhl et al., Astrophys. J. 599, 786 (2003); T. J. Pearson et al., Astrophys. J. 591, 556 (2003); P. F. Scott et al., Mon. Not. R. Astron. Soc. 341, 1066 (2003).

[14] M. Tegmark et al., Astrophys. J. 606, 702 (2004).

[15] R. Sanders, Mon. Not. R. Astron. Soc. 342, 901 (2003). 